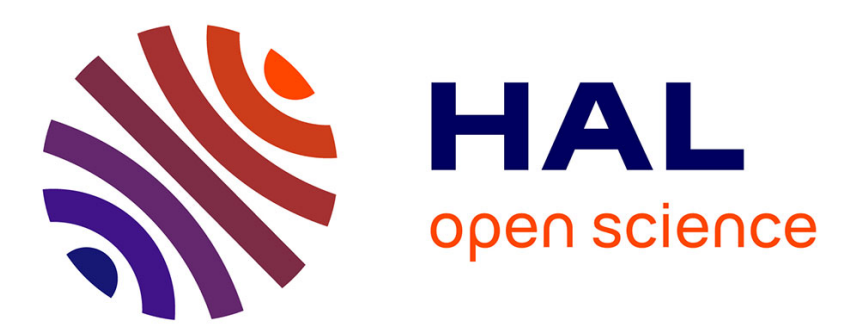

\title{
Graphical solution for system optimum dynamic traffic assignment with day-based incentive routing strategies
}

Chuan-Lin Zhao, Ludovic Leclercq

\section{To cite this version:}

Chuan-Lin Zhao, Ludovic Leclercq. Graphical solution for system optimum dynamic traffic assignment with day-based incentive routing strategies. Transportation Research Part B: Methodological, 2018, 117 (Part A), pp87-100. 10.1016/j.trb.2018.08.018 . hal-01873677

\section{HAL Id: hal-01873677 \\ https://hal.science/hal-01873677}

Submitted on 21 Jan 2019

HAL is a multi-disciplinary open access archive for the deposit and dissemination of scientific research documents, whether they are published or not. The documents may come from teaching and research institutions in France or abroad, or from public or private research centers.
L'archive ouverte pluridisciplinaire HAL, est destinée au dépôt et à la diffusion de documents scientifiques de niveau recherche, publiés ou non, émanant des établissements d'enseignement et de recherche français ou étrangers, des laboratoires publics ou privés. 


\title{
Graphical solution for system optimum dynamic traffic assignment with day-based incentive routing strategies
}

\author{
Chuan-Lin Zhao ${ }^{\mathrm{a}, \mathrm{b}, *}$, Ludovic Leclercq ${ }^{\mathrm{a}}$ \\ ${ }^{a}$ Univ. Lyon, ENTPE, IFSTTAR, LICIT, F-69518, Lyon, France \\ ${ }^{b}$ School of Civil and Transportation Engineering, Beijing University of Civil Engineering and \\ Architecture, Beijing 100044, China
}

\begin{abstract}
This paper analyzes the dynamic traffic assignment problem on a three-alternative network with day-based incentive routing strategies by using graphical solution method. It is assumed that the cumulative count curve of vehicles is known and that the arrival rate is unimodal. The dynamic system optimum (DSO) allocation lines are first drawn based on calculus of variations. Three possible optimal allocation lines are analyzed. A day-based incentive routing strategy is designed and conditions that when and how to implement the incentive scheme to realize DSO are then derived. Extension to general parallel networks is also given. Examples are presented to demonstrate the effectiveness of the scheme.
\end{abstract}

Keywords: System optimum, Dynamic traffic assignment, Graphical solution method, Incentive strategy

\section{Introduction}

Until now, many methods have been proposed to reduce the traffic congestion in both static and dynamic cases, including (1) economic policies, e.g., congestion pricing (Pigou, 1920; Evans, 1992; Yang and Huang, 2005; Laval et al., 2015; Daganzo and Lehe, 2015); tradable credits (Yang and Wang, 2011; Akamatsu and Wada, 2017; Lahlou and Wynter, 2017); rewards (Rouwendal et al., 2012); transit subsidy (Parry and Small, 2009); parking pricing (D'Acierno et al., 2006); and (2) engineering control schemes, e.g., speed limits (Knoop et al., 2010; Yang et al., 2012; Chen and Ahn, 2015); ramp metering (Papageorgiou and Kotsialos, 2002); license plate rationing (Nie, 2017); lane control (Dahlgren, 1998; Daganzo and Cassidy, 2008; Fosgerau, 2011) and so on. Although the popular congestion pricing schemes have been implemented in some cities, there are still a long way toward eliminating the public's reluctance to accept tolls. For tradable credits, to our best knowledge, it has not been implemented in any cities yet.

\footnotetext{
${ }^{*}$ Corresponding author. Tel. : +86 18810638340

Email addresses: zhaochuanlin@bucea.edu.cn (Chuan-Lin Zhao), ludovic.leclercq@entpe.fr (Ludovic Leclercq)
} 
For engineering control schemes, most studies investigate the effects of these schemes on network performance based on simulation approach. Other combined schemes (e.g., Daganzo, 1995; Daganzo et al., 2002; Basso and Jara-Díaz, 2012; Wang et al., 2015) are also studied in literature.

For this paper we focus on the day-based incentive routing strategies that should be associated with multiple days, which is a very challenging research topic. Take two routes for example. One route is the main route $M$ with lower free flow travel time. Another route is side route $S$ with higher free flow travel time. Users who want to choose $M$ for one-day use need to pay some credits or points which can be freely obtained by choosing $S$ for a few days. Note that the concept of credit in this paper is different with the one in Yang and Wang(2011) in the following two aspects: (1)in Yang and $\operatorname{Wang}(2011)$ the credits that are freely obtained from the government are tradable. Users need to pay some money to obtain additional credits or can earn some money by selling some credits. That is, the value of the credits as one part of total trip cost will affect the users' route choice decisions; (2)while in this paper the credits or points that represent one kind of rights are freely obtained by choosing side route. With the credits or points users have the right to choose the main route. That is, the only role of the credits or points is to encourage or motivate users to choose the side route. Note that the credits are not tradable in this paper. Thus the values of the credits are not considered as one part of the route trip cost. For each user, they have to make the decisions, i.e., how many days in one period (e.g., $P$ days) I can choose route $M$ and how many days in one period I need to choose route $S$ ? The question of interest is how to design this day-based incentive routing strategy to realize the state of system optimum, which will be investigated in this study. That is, the whole idea is to obtain a daily flow pattern, which is close to SO while users keep follow a UE discipline. The switch is made considering day-based incentive, i.e., by providing credits for not using the optimal individual path for some days.

Another related literature to this study is about system optimum dynamic traffic assignment(SODTA) problem. A vast body of literature has been developed in this area over the past four decades (e.g., Merchant and Nemhauser,1978a,b; Friesz et al., 1989; Ghali and Smith, 1995; Ziliaskopoulos, 2000; Nie, 2011; Abdul Aziz and Ukkusuri, 2012; Carey and Watling, 2012; Zhu and Ukkusuri, 2013; Ma et al., 2014; Doan and Ukkusuri, 2015; Lu et al., 2016). Two kinds of models, i.e., discrete-time models and continuous-time models, are mostly used in the existing literature. Most discrete-time models share a similar mathematical programming structure with heuristic approaches to find the DSO solutions; while the continuous-time models use continuous optimal control method.

A persistent issue is the need to trade-off mathematical tractability with traffic realism. This paper will focus on another analytical method, i.e., graphical solution method, to find the exact DSO solutions. Note that this method belongs to continuoustime models. There are only a few publications that have approached the DSO problem by using this method. To our best knowledge, Muñoz and Laval (2006) are the first to introduce the method based on dynamic optimality conditions and calculus of varia- 
tions to draw the dynamic system optimum (DSO) allocation lines. Recently, Laval et al.(2015) analyzed the effects of system optimum tolls on dynamic traffic assignment problem in a two-alternative network. As mentioned in the above two papers, the time intervals when the alternatives are used at capacity can be defined uniquely, but the allocation of the queues is not. Laval (2009) also studied the dynamic user optimum (DUO) traffic assignment problem in a simple parallel network and derived some analytical results. One important assumption is used in the above three papers that the cumulative count curve of vehicles is known and exogenous. That is, no departure time choice is considered in these papers. By relaxing the assumption, Arnott et al. (1990a) and Shen and Zhang (2009) studied both departure time and route choice problem in a parallel network.

In all, in this study we will investigate the DSO traffic assignment problem in a three-alternative network with the assumption that the cumulative arrival curve of vehicles is known based on Muñoz and Laval (2006). The logic framework of this paper includes three parts, i.e., Step a: Solve the DSO solutions by using graphical solution method; Step b: Design the routing strategies based on the DSO solutions and prove the DSO is DUE with the designed strategies; Step c: Extension to general parallel networks.

This rest of the paper is organized as follows: Section 2 presents the modeling assumptions and problem formulation in a three-alternative network. Section 3 illustrates the extended graphical solution method to draw the DSO allocation lines. Section 4 describes the day-based incentive routing strategies and examines the DUO equilibrium state obtained as a result of its application. Extension to general parallel networks is also given in this section, and finally Section 5 concludes the paper.

\section{Problem formulation}

The network consists of one origin, one destination, and three alternatives (i.e., a freeway with two lanes and a city-street); see Fig.1. Note that the network, i.e., one-lane freeway with city-street alternative, is often used to analyze classical morning commute problem, but here we add the opportunity to consider the lane-allocation strategy on the freeways, especially to analyze the effects of day-based incentive routing strategies. Suppose that we know the cumulative count curve $A(t)$ of vehicles entering a freeway segment with two lanes or city-street with longer free flow travel time. Let the corresponding flow be $\lambda(t)=\dot{A}(t)$. As shown in Fig.1, the cumulative count curve of vehicles at time $t$ using route $r$ is denoted $A_{r}(t)$ and the flow, $\lambda_{r}(t)=\dot{A}_{r}(t)$. Clearly, we have $\lambda(t)=\lambda_{2}(t)+\lambda_{1}(t)+\lambda_{0}(t)$. Another assumption is used throughout the paper that the arrival pattern is unimodal, where the arrival rate continuously increases to exceed total capacities of the three routes, and then decreases.

Point queue approach will be used to model the dynamic route choice problem. The capacity and free flow travel time of each alternative $r, r=2,1,0$, are denoted $\mu_{r}$ and $\tau_{r}$, respectively. Suppose that the three bottlenecks are located at the beginning of the three alternatives. Let $\tau_{r}(t)$ be the trip time in route $r$ experienced by a user arriving 
at time $t$ :

$$
\tau_{r}(t)=\tau_{r}+w_{r}(t),
$$

where $w_{r}(t)$ is the queuing delay, which can be expressed as (see Arnott et al., 1990b; Laval et al., 2015):

$$
w_{r}(t)=\frac{A_{r}(t)-A_{r}\left(t_{r}\right)}{\mu_{r}}-\left(t-t_{r}\right), t_{r} \leqslant t \leqslant T_{r},
$$

where $t_{r}$ and $T_{r}$ represent the times when route $r$ begins and ends being congested, respectively.

Due to the departure time of each user is fixed, the goals in this paper are: (1) to determine the time dependent path flow distributions that vehicles should follow so that the total time spend in the system is minimized; (2) to design the day-based incentive routing strategy based on the flow diversion results to realize the DSO.

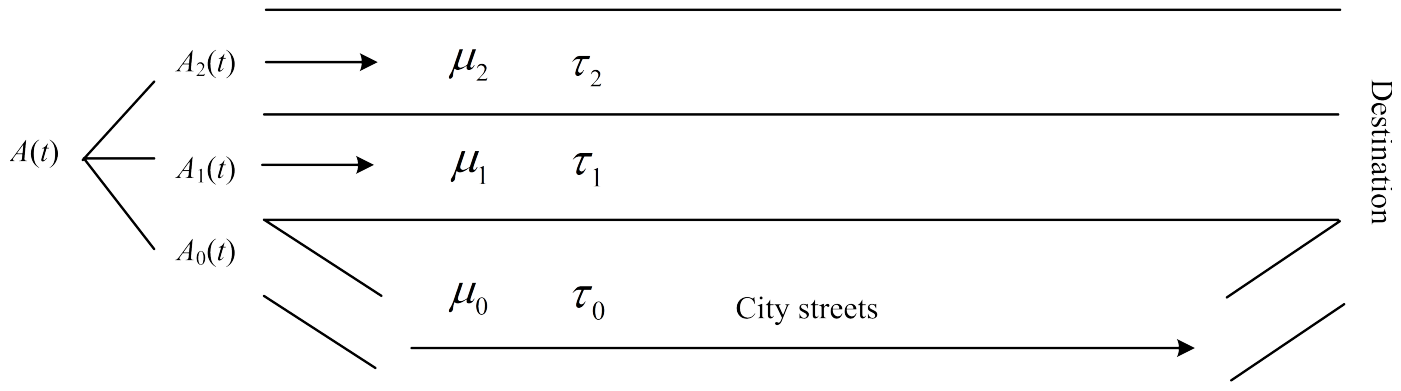

Fig. 1. Schematic of the three route network.

\section{One method to draw the DSO allocation lines}

In this section, we will draw the DSO allocation lines based on graphical solution method and calculus of variations. Note that "the allocation line" means the determined time intervals when flows should be diverted to different routes. More details about this kind of line will be given in Subsections 3.1 and 3.2 when drawing these lines. Before we draw the lines, optimality conditions should be given first. As mentioned in Muñoz and Laval (2006), the route with the least marginal cost will be chosen for all times and all origins. For a given route the marginal cost corresponds to the extra delay imposed by an additional vehicle on all following vehicles (Specifically, the extra delay imposed by an additional vehicle at time $t$ is equal to the duration until the queue vanishes; see Ghali and Smith(1995) and Kuwahara(2007) for more details) plus the additional vehicle's trip time.

In the following analysis, we consider the case $\tau_{2}<\tau_{1}<\tau_{0}{ }^{1}$. Next we will first review the graphical solution method introduced in Muñoz and Laval (2006), and then

\footnotetext{
${ }^{1}$ Actually, case $\tau_{1}<\tau_{2}<\tau_{0}$ is similar with this case. Because we just need to exchange the positions of " 1 " and " 2 " when drawing the allocation lines. Note that for the third case $\tau_{2}=\tau_{1}<\tau_{0}$, we just need to think of Route 2 and Route 1 as one route with capacity $\mu_{2}+\mu_{1}$.
} 
develop our method. Three cases will be considered. Case 1: $\left(\mu_{2} ; \mu_{1} ; \mu_{0}\right)=\left(\mu_{2} ; \infty ; \infty\right)$; Case 2: $\left(\mu_{2} ; \mu_{1} ; \mu_{0}\right)=\left(\mu_{2} ; \mu_{1} ; \mu_{0}\right)$; Case $3:\left(\mu_{2} ; \mu_{1} ; \mu_{0}\right)=\left(\mu_{2} ; \mu_{1} ; \infty\right)$, where $\mu_{r}$ represents the value of bottleneck capacity on route $r$ is constant. The first two cases have been considered in Muñoz and Laval (2006). We will extend the graphical solution method in Case 3.

\subsection{Review of Muñoz and Laval's method}

\subsubsection{Case 1: $\left(\mu_{2} ; \mu_{1} ; \mu_{0}\right)=\left(\mu_{2} ; \infty ; \infty\right)$}

Due to the unlimited capacities of routes 1 and 0 , these two routes will never become congested. Then, at optimum, only route 2 and route 1 will be used in this case. Let $T_{2}$ be the moment when the queue of route 2 vanishes and $T_{1}$ the moment when the last vehicle is assigned to route 1 . The optimality condition, in which the sum of the quantities of variations is zero, is given by

$$
T_{2}-T_{1}=\tau_{1}-\tau_{2}
$$

For more details about the condition, see Muñoz and Laval (2006).

The DSO solution for this case is presented in Fig.2. Note that $t_{2}$ is the moment when $\lambda(t)$ initially exceeds $\mu_{2}$. Based on the optimality condition of Equation (3), as mentioned in Muñoz and Laval (2006) and Laval et al.(2015), as long as the arrival rate is lower than $\mu_{2}$, there is no need for re-routing. Re-routing starts immediately when $\lambda(t)>\mu_{2}$ (i.e., at $t_{2}$ ) and ends at $T_{1}$. The optimal solution implies no queue at the bottleneck $\mu_{2}$ during re-routing. Congestion happens on route 2 after the rerouting period between $T_{1}$ and $T_{2}$. Graphically, as shown in Fig.2(a), starting from point $\left(t_{2}, A\left(t_{2}\right)\right)$, draw the black line that grows linearly at a rate $\mu_{2}$. $A_{2}(t)$, the green line as shown in Fig.2(b), can be determined by shifting the arrival curve $A(t)$ down vertically until the horizontal distance between the intersection points with black line equals $\tau_{1}-\tau_{2}$. $A_{1}(t)$, the green line as shown in Fig.2(c), can be determined by $A_{1}(t)=A(t)-A_{2}(t)$ when $t_{2} \leqslant t \leqslant T_{1}$.

We call the red line $L(t)$ in Fig.2(a) as "allocation line". That is, this line can determine the time interval, i.e., $\left(T_{1}, T_{2}\right)$, and then determine the total time intervals, i.e., $\left(0, t_{2}\right),\left(t_{2}, T_{1}\right)$ and $\left(T_{1}, T_{2}\right)$, when to assign flows to different routes to realize DSO. In all, the DSO assignment for users arriving at time $t$ satisfy:

1. $0 \leqslant t \leqslant t_{2}$ : everybody uses route 2 .

2. $t_{2} \leqslant t \leqslant T_{1}$ : the route 2 is used at capacity, excess inflow uses the route 1 .

3. $t \geqslant T_{1}$ : everybody uses the route 2 .

Remark 1: The above analysis for Case 1 can also be used to analyze Case 1', i.e., $\left(\mu_{2} ; \mu_{1} ; \mu_{0}\right)=\left(\mu_{2} ; \infty ; \mu_{0}\right)$. Clearly, no users want to choose route 0 because route 1 with unlimited capacity has lower free flow travel time than route 0. 


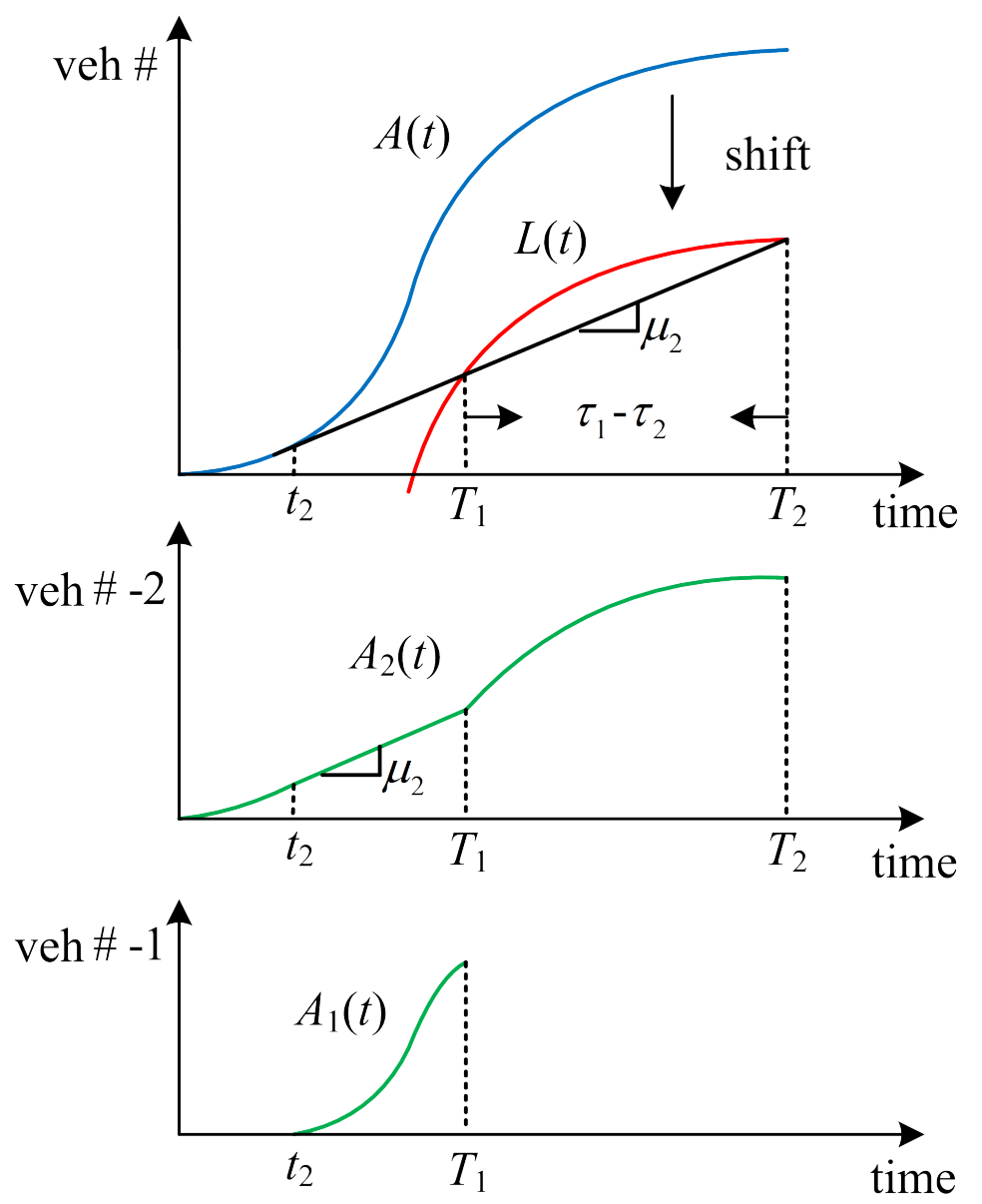

(a)

(b)

(c)

Fig. 2. Optimal solution for Case 1: (a) The procedure of drawing allocation lines. (b) DSO arrival curve of route 2. (c) DSO arrival curve of route 1.

\subsubsection{Case 2: $\left(\mu_{2} ; \mu_{1} ; \mu_{0}\right)=\left(\mu_{2} ; \mu_{1} ; \mu_{0}\right)$}

As analyzed in Muñoz and Laval (2006), the optimality conditions for this case are given by

$$
\left\{\begin{array}{l}
T_{2}-T_{1}=\tau_{1}-\tau_{2} \\
T_{1}-T_{0}=\tau_{0}-\tau_{1}
\end{array}\right.
$$

where $T_{r}, r=1,0$, represents the moment when the last vehicle leaves the bottleneck of route $r$, and $T_{2}$ is the moment when the queue of route 2 vanishes.

The DSO graphical solution for this case is presented in Fig.3. Three steps are needed to draw the allocation lines as shown in Fig.3(a).

Step 12: Lengthen $A(t)$ horizontally; see the horizontal dotted line in Fig.3(a).

\footnotetext{
${ }^{2}$ It is worth noting that Step 1 is necessary to make sure that the queue can be cleared and the optimality conditions are satisfied. Actually, for Case 1 we also need this step to obtain the optimal solution; see Fig.4 for example. Of course, we do not need to do so if the initial $A(t)$ is long enough.
} 
Notice that the extension line is still one part of $A(t)$.

Step 2: Choose one point $M$ on $A(t)$ with large enough time value (e.g., $T_{M}$ ) as starting point, draw the initial allocation line $L(t)$ (the right red line in Fig.3(a)) based on the optimality conditions of Equation (4). Note that the slopes of the three parts of the line $L(t)$, from up to down, are $\mu_{2}, \mu_{2}+\mu_{1}, \mu_{2}+\mu_{1}+\mu_{0}$, respectively.

Step 3: Shift $L(t)$ from the right to the left along $A(t)$ until it "touches" $A(t)$ again (the left red line in Fig.3(a)). Two intersection points are $\left(T_{2}, A\left(T_{2}\right)\right)$ and $\left(t_{0}, A\left(t_{0}\right)\right)$. Due to the assumption that the arrival rate continuously increases to exceed total capacities of the three routes, all of the three routes could possibly be used to divert vehicles in this case. That is, there exists $t=t_{0}$, it holds $\lambda\left(t_{0}\right)=\mu_{2}+\mu_{1}+\mu_{0}$. Note that $t_{0}$ and $T_{0}$ will coincide when $t_{0}+\tau_{0}-\tau_{1}=T_{1}$ as mentioned in Muñoz and Laval (2006).

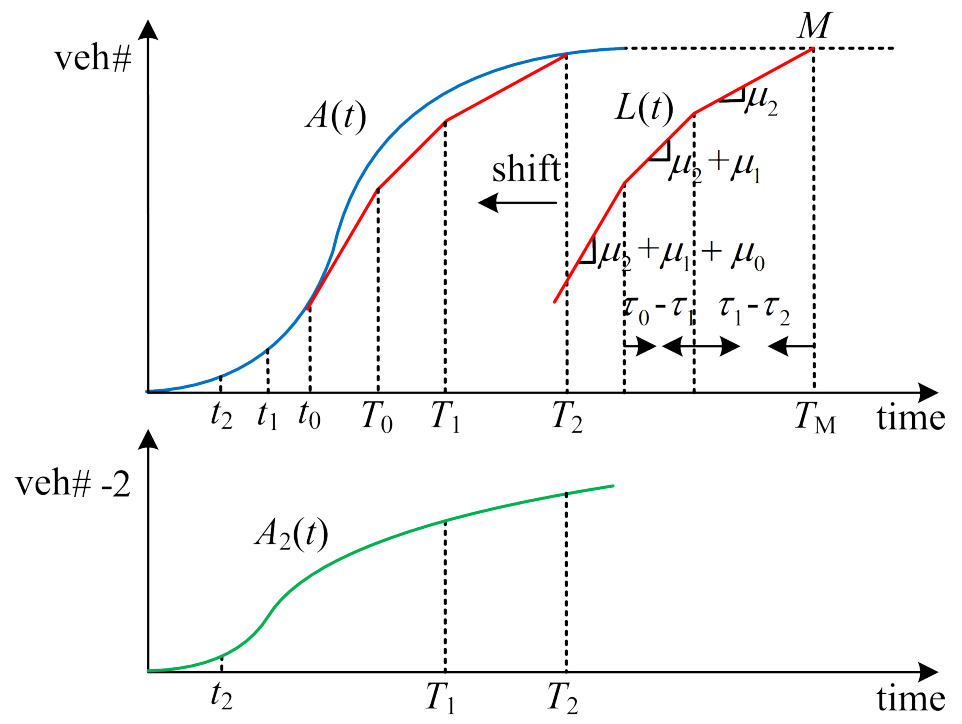

(a)

(b)

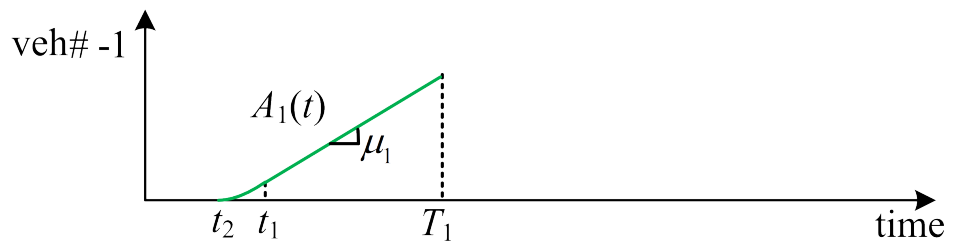

(c)

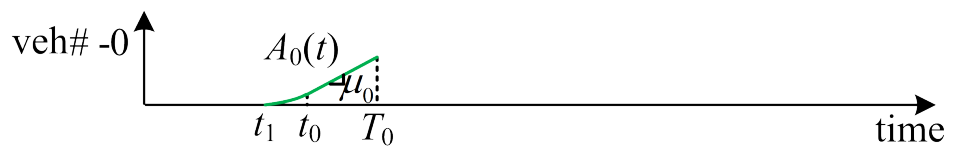

Fig. 3. One extreme optimal solution for Case 2: (a) The procedure of drawing allocation lines. (b) DSO arrival curve of route 2. (c) DSO arrival curve of route 1. (d) DSO arrival curve of route 0.

Note that in Fig.3(a), $\lambda\left(t_{2}\right)=\mu_{2}$ and $\lambda\left(t_{1}\right)=\mu_{2}+\mu_{1}$. In all, with this procedure, based on the allocation lines the DSO assignment for users arriving at time $t$ satisfy:

1. $0 \leqslant t \leqslant t_{2}$ : everybody uses route 2 .

2. $t_{2} \leqslant t \leqslant t_{1}$ : the route 2 is used at capacity, excess inflow uses the route 1 . 


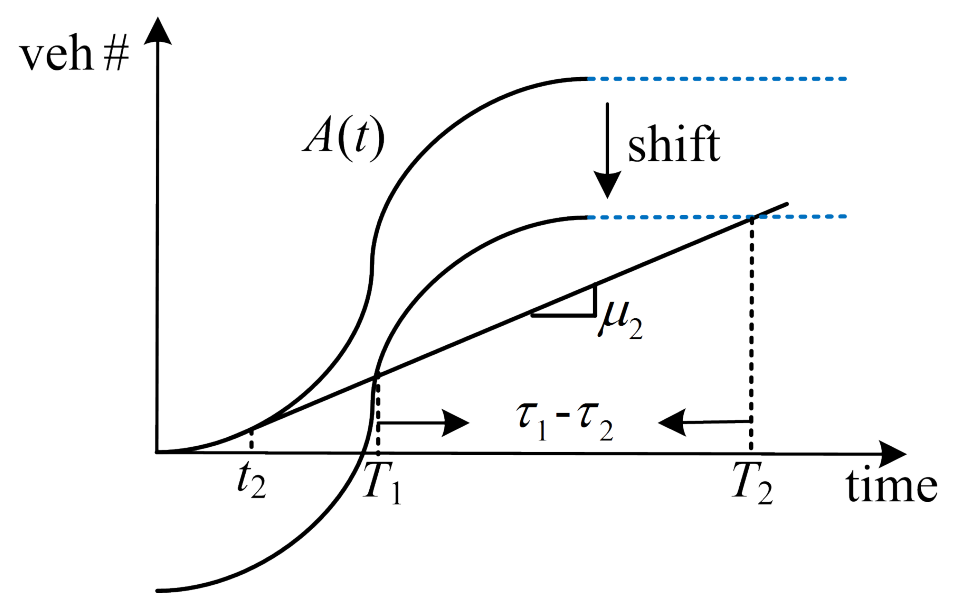

Fig. 4. One example to illustrate Step 1 for Case 1.

3. $t_{1} \leqslant t \leqslant t_{0}$ : the route 2 and route 1 are used at capacity, excess inflow uses the route 0 .

4. $t_{0} \leqslant t \leqslant T_{0}$ : all of the three routes are used at capacity.

5. $T_{0} \leqslant t \leqslant T_{1}$ : both routes, route 2 and route 1 , are used at capacity.

6. $t \geqslant T_{1}$ : everybody uses the route 2 .

We call the two red lines $L(t)$ in Fig.3(a) as "allocation lines", based on which $T_{r}$, $r=2,1,0$, is uniquely identified for any optimal solution and time intervals in which time the routes will be used are also uniquely determined. As mentioned in Muñoz and Laval (2006) and Laval et al.(2015), in $t_{0} \leqslant t \leqslant T_{0}$ the bottleneck of route 0 is working at capacity. In $t_{1} \leqslant t \leqslant T_{1}$ the bottleneck of route 1 is working at capacity. In $t_{2} \leqslant t \leqslant T_{2}$ the bottleneck of route 2 is working at capacity. But the assignment solution between $t_{0}$ and $T_{2}$ in terms of the route-specific flows $\lambda_{r}(t), r=2,1,0$ is not unique. Fig.3(b), (c) and (d) show one extreme optimal solution of $A_{r}(t)$ (i.e., green lines in Fig.3) based on the Fig.6(b) in Muñoz and Laval (2006). With this solution no queue grows on routes 1 and 0 . Table 1 gives the detailed optimal solution in terms of arrival rate.

Table 1. One extreme optimal solution based on Fig.3

\begin{tabular}{llll}
\hline Time interval & $\lambda_{2}(t)$ & $\lambda_{1}(t)$ & $\lambda_{0}(t)$ \\
\hline $0 \leqslant t \leqslant t_{2}$ & $\lambda(t)$ & 0 & 0 \\
$t_{2} \leqslant t \leqslant t_{1}$ & $\mu_{2}$ & $\lambda(t)-\mu_{2}$ & 0 \\
$t_{1} \leqslant t \leqslant t_{0}$ & $\mu_{2}$ & $\mu_{1}$ & $\lambda(t)-\mu_{2}-\mu_{1}$ \\
$t_{0} \leqslant t \leqslant T_{0}$ & $\lambda(t)-\mu_{2}-\mu_{1}$ & $\mu_{2}$ & $\mu_{1}$ \\
$T_{0} \leqslant t \leqslant T_{1}$ & $\lambda(t)-\mu_{2}$ & $\mu_{2}$ & 0 \\
$t \geqslant T_{1}$ & $\lambda(t)$ & 0 & 0 \\
\hline
\end{tabular}




\subsection{Our method}

In this subsection, we consider the Case $3:\left(\mu_{2} ; \mu_{1} ; \mu_{0}\right)=\left(\mu_{2} ; \mu_{1} ; \infty\right)$, and introduce an extended method (i.e., shift $L(t)$ from the left to the right) to draw the allocation lines, which is different from the above two methods (i.e., shift $A(t)$ from up to down for case 1; shift $L(t)$ from the right to the left for case 2 ). The optimality conditions for this case are the same with Case 2; see Equations (4). With this extended method, three steps are needed to draw the allocation lines as shown in Fig.5.

Step 1: Lengthen $A(t)$ horizontally.

Step 2: Starting from the point $\left(t_{1}, A\left(t_{1}\right)\right)$, where $\lambda\left(t_{1}\right)=\mu_{2}+\mu_{1}$, draw the initial allocation line $L(t)$ (i.e., the left or down red line in Fig.5) based on the optimality conditions. Note that the slopes of the two parts of the line, from the left to the right, are $\mu_{2}+\mu_{1}, \mu_{2}$, respectively.

Step 3: Shift $L(t)$ from the left to the right along $A(t)$ until the end point of the line "touches" $A(t)$ again (the right or up red line in Fig.5).

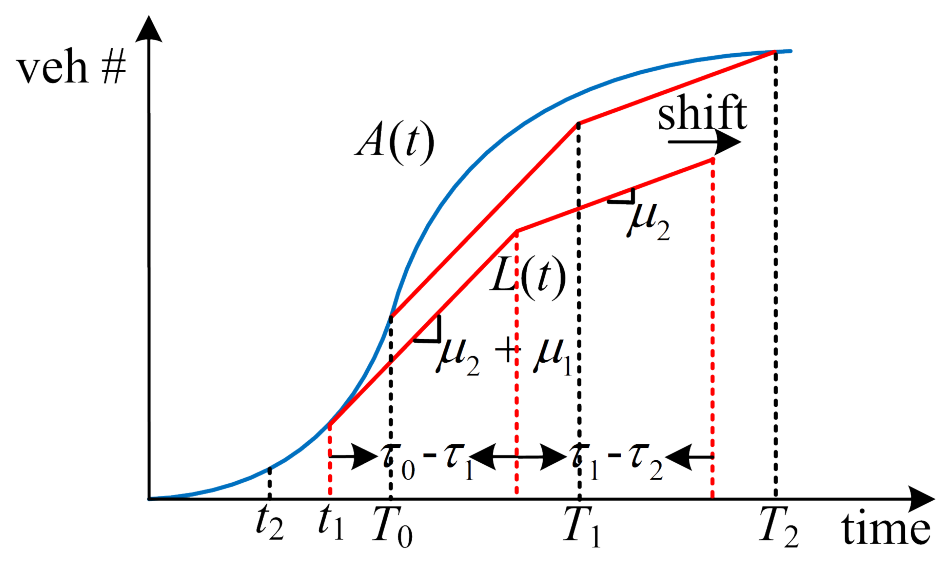

Fig. 5. One possible optimal solution for Case 3.

We call the red lines $L(t)$ in Fig.5 as "allocation lines", based on which the DSO assignment for users arriving at time $t$ satisfy:

1. $0 \leqslant t \leqslant t_{2}$ : everybody uses route 2 .

2. $t_{2} \leqslant t \leqslant t_{1}$ : the route 2 is used at capacity, excess inflow uses the route 1 .

3. $t_{1} \leqslant t \leqslant T_{0}$ : the route 2 and route 1 are used at capacity, excess inflow uses the route 0 .

4. $T_{0} \leqslant t \leqslant T_{1}$ : both routes, the route 2 and route 1 are used at capacity.

5. $t \geqslant T_{1}$ : everybody uses the route 2 .

It is worth noting that Fig.5 only shows one possible optimal solution for Case 3. There exist two other possible allocation lines; see Fig.6 and Fig.7 for example. For convenience, three key points on the allocation line as shown in Fig.6 are denoted as $K_{1}, K_{2}, K_{3}$, respectively. Perform the Step 3 in Subsection 3.2, when the point $K_{3}$ first touches $A(t)$, there exist three possible positions for point $K_{2}$ : 
(i) Under $A(t)$ as shown in Fig.5.

(ii) On $A(t)$ as shown in Fig.6.

(iii) Above $A(t)$ as shown in Fig.7(a).

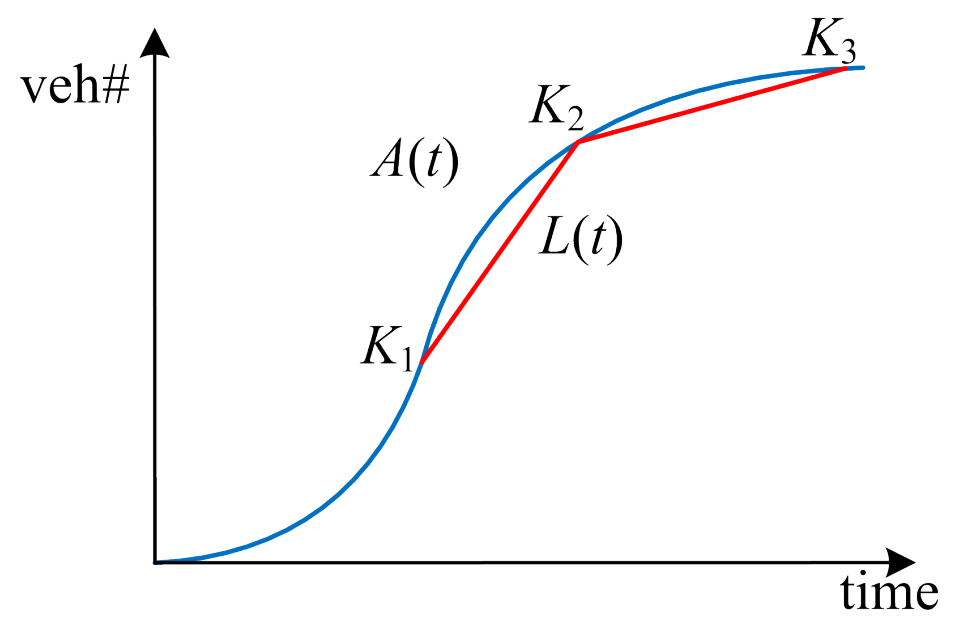

Fig. 6. The second possible optimal solution for Case 3 .

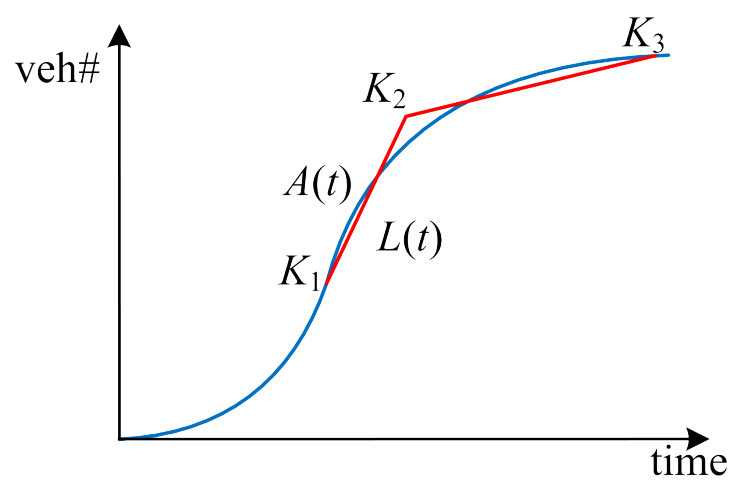

(a)

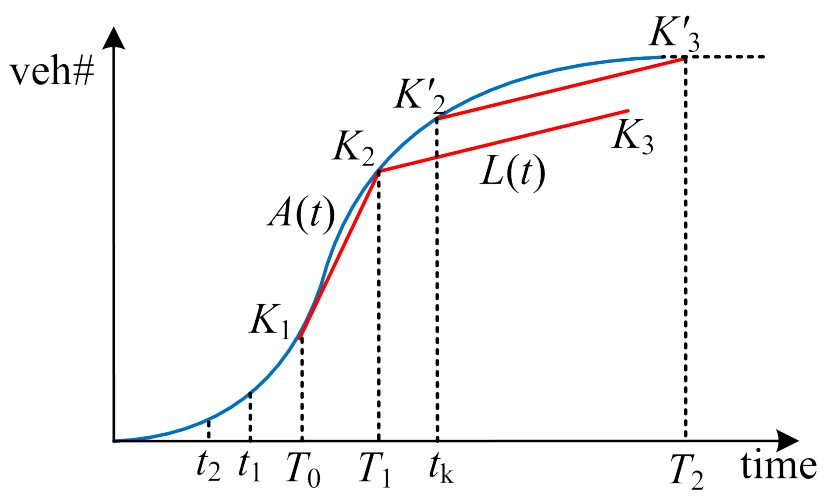

(b)

Fig. 7. The third possible optimal solution for Case 3: (a) One example of the position of $K_{2}$ that is above $A(t)$ when $K_{3}$ touches $A(t)$; (b) The procedure to draw allocation line.

Here we focus on the analysis of Case (iii). Actually the analysis for Case (ii) is the same with Case (i). As shown in Fig.7(b), the procedure to draw the allocation lines includes:

Step 1: Lengthen $A(t)$ horizontally.

Step 2: The same procedure to draw $L(t)$ in Fig.5.

Step 3: From the left to the right, shift $L(t)$ along $A(t)$ until $K_{2}$ touches $A(t)$. Clearly, $K_{3}$ is still under $A(t)$. 
Step 4: To satisfy the optimality condition, starting from $K_{2}$, shift the right part of $L(t)$ (i.e., line segment $K_{2} K_{3}$ in Fig.7(b)) along $A(t)$ until $K_{3}$ touches $A(t)$. As shown in Fig.7(b), the two new intersection points are denoted as $K_{2}^{\prime}$ and $K_{3}^{\prime}$, respectively.

With this procedure, $t_{k}$ in Fig.7(b) is uniquely defined corresponding to the point $K_{2}^{\prime}$ when comparing with Fig.5. Based on the allocation lines as shown in Fig.7(b), the DSO assignment for users arriving at time $t$ satisfy:

1. $0 \leqslant t \leqslant t_{2}$ : everybody uses route 2 .

2. $t_{2} \leqslant t \leqslant t_{1}$ : the route 2 is used at capacity, excess inflow uses the route 1 .

3. $t_{1} \leqslant t \leqslant T_{0}$ : the route 2 and route 1 are used at capacity, excess inflow uses the route 0 .

4. $T_{0} \leqslant t \leqslant T_{1}$ : both the route 2 and route 1 are used at capacity.

5. $T_{1} \leqslant t \leqslant t_{k}$ : the route 2 is used at capacity, excess inflow uses the route 1 . This is because the condition, i.e., $\mu_{2}<\lambda(t)<\mu_{2}+\mu_{1}$, holds when $T_{1} \leqslant t \leqslant t_{k}$ as shown in Fig.7(b).

6. $t \geqslant t_{k}$ : everybody uses the route 2 .

Note that the procedure as shown in Fig.7 can make sure that the solutions are system optimal. Firstly, it is worth noting that the point $K_{2}$ cannot be above $A(t)$ as shown in Fig.7(a). This is because the cumulative departure curve cannot be higher than the cumulative arrival curve at each time $t$. Secondly, we check the change of the system total trip times by considering the diversion of one more or one fewer vehicle to route 0 at each time $t$, i.e., the variational principle for optimality. The optimality of this solution can be checked by calculus of variations as follows.

First consider the diversion of one more vehicle to route 0 .

If $t \leqslant t_{2}$ then the diversion has no impact on the freeway queue since the queue would be triggered at the same time. However, the diversion imposes an extra delay of $\tau_{0}-\tau_{2}$ on the diverted vehicle.

If $t_{2} \leqslant t \leqslant T_{0}$ then the diversion imposes an extra delay of at least $\tau_{0}-\tau_{1}$ or at most $\tau_{0}-\tau_{2}$ on the diverted vehicle.

If $T_{0}<t \leqslant T_{1}$ then (1) when the queue is assigned to route 1 , the system benefits (i.e., $T_{1}-t$ ) include two parts, the queue would vanish at most $1 / \mu_{1}$ units of time earlier and the diverted vehicle would save its freeway delay. However, the diverted vehicle would suffer an extra delay of at least $\tau_{0}-\tau_{1}$. Since $T_{1}-t<\tau_{0}-\tau_{1}$, diverting this vehicle is not optimal. Since at $T_{1}$ the freeway queue vanishes, diverting the vehicle does not affect the formation of the second queue; (2) when the queue is assigned to route 2, we have the similar result.

If $t>T_{1}$ then the case is similar with Case 1 and the optimality condition (i.e., Eq.3) need to be satisfied.

Now consider the diversion of one fewer vehicle at $t$ to route 0 . We can get the similar results. That is, this perturbation is also non-optimal. For more details about the analysis, please refer to Section 3.2 in Muñoz and Laval (2006).

Remark 2: Our method for Case 3, i.e., move $L(t)$ from the left to the right along $A(t)$, can be used to analyze Case 1 to find the optimal solution. But the method of 
Muñoz and Laval (2006) for Case 1 can not be used for Case 3. This is because the optimality conditions of Case 3 can not be satisfied when shifting $A(t)$ vertically from up to down. Furthermore, our method with a modification can also be used for Case 2, i.e., "shift $L(t)$ along $A(t)$ " should be modified to "shift $L(t)$ along the lines with the slope of $\mu_{2}+\mu_{1}+\mu_{0}$ "; see Fig. 8 for more details. As shown in Fig.8, we first need to draw the line $k_{1}$ with the slope of $\lambda\left(t_{0}\right)=\mu_{2}+\mu_{1}+\mu_{0}$ that passes through the point $\left(t_{0}, A\left(t_{0}\right)\right)$ and choose the point $\left(t_{0}, A\left(t_{0}\right)\right)$ on the line $k_{1}$, i.e., $K_{2}$, as starting point; and then draw the allocation line, i.e., line segments $K_{2} K_{3}$ and $K_{3} K_{4}$, based on the optimality conditions of Equations (4); and at last move the line $K_{2} K_{3} K_{4}$ from the left to the right along $k_{1}$ until $K_{4}$ touches $A(t)$. Also note that in the process of moving line segments $K_{2} K_{3} K_{4}$, the position of the point $K_{3}$ may be under (or on, or above) $A(t)$ as mentioned in the previous analysis. Our method can also be extended to analyze the general parallel networks, especially to determine the highest number of routes that could possibly be used to divert vehicles, which will be discussed in the last section of the paper.

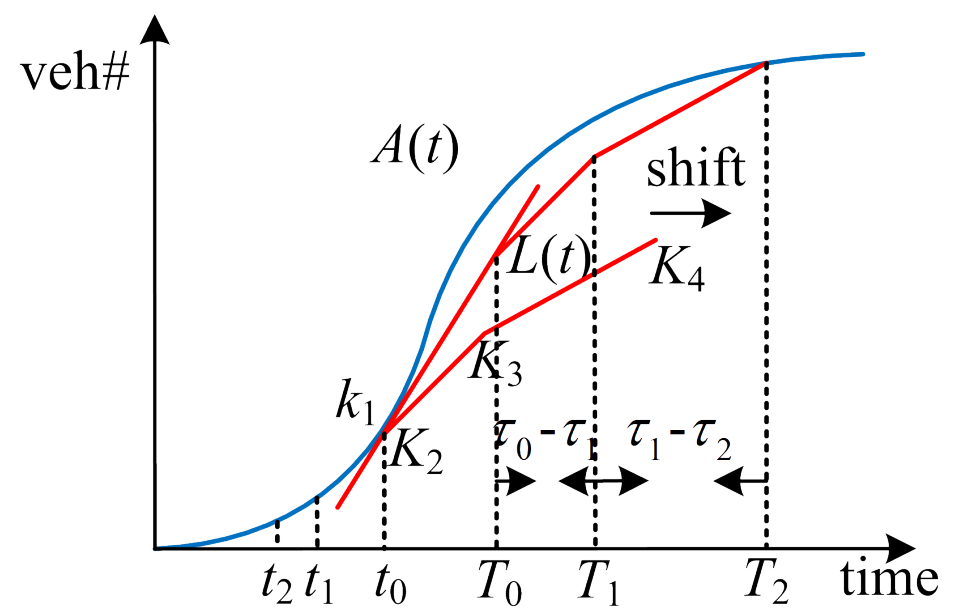

Fig. 8. Graphical optimum solution for Case 2 based on our modified method.

In summery, in this section we uniquely defined the time intervals at which time which route will be used at the state of DSO by drawing the graphical allocation lines. But the uniqueness of the flow assignment results can not be guaranteed when two or more routes are used at capacity. It is shown that at DSO route 2 with lowest free flow travel time is always being used and route 1 is used for less time and route 0 is used for least time. Based on these results, in the next section a day-based incentive routing strategy will be designed to realize the state of DSO.

\section{Day-based incentive routing strategy}

In this section, we investigate the long-term dynamic route choice behavior and design one kind of day-based incentive routing strategy to realize the state of DSO which is obtained by graphical solution method. 
Users are assumed to be perfectly rational and want to minimize their own travel times every day when making route choice decisions. Basically, user follows a double layer optimization principle. Over the long run, e.g., one period of $P$ days, they may choose to take non optimal route to gain credits and be able to spend them on the fastest track some days. Over the short run (daily based), they are following a UE principle by trying to minimize their travel time. As a result, we use the term of PUE (i.e., Period User Equilibrium) to distinguish the traditional within-day DUE.

\subsection{Definition and properties of the strategy}

Inspired by Daganzo and Garcia (2000), in one period (e.g., $P$ days), the fraction of days that a commuter arriving at time $t$ choosing route $r, r=2,1,0$, is denoted as $f_{r}(t)$. Clearly, it holds $f_{2}(t)+f_{1}(t)+f_{0}(t)=1$. Let $f(t)=\left(f_{r}(t), r=2,1,0\right)$ be the fraction vector. Before we describe the routing strategy, the concept of Period User Equilibrium (PUE) or Period User Optimum (PUO) should be given first: at PUE, the total trip times of $P$ days are minimized for each commuter arriving at time $t$. Based on this, at PUE the fraction of days for one commuter choosing route $r, r=2,1,0$, is denoted as $f_{r}^{u}(t)$. Let $f^{u}(t)=\left(f_{r}^{u}(t), r=2,1,0\right)$ be the PUE fraction vector.

Our goal is to design a day-based incentive routing strategy to make sure that the DSO is PUE with this strategy. Let $f^{s}(t)=\left(f_{r}^{s}(t), r=2,1,0\right)$ (where $f_{r}^{s}(t)$ denotes at DSO the fraction of total flows arriving at time $t$ that are assigned to the route $r$, which can be obtained by graphical solution method) be the DSO fraction vector. The DSO allocation lines analyzed in the above section provide a powerful tool to design this strategy. Specifically, what we should do is (1) to design a scheme to realize DSO based on the graphical system optimal solution and (2) to prove that DSO is PUE under this scheme, i.e., equations $f_{r}^{s}(t)=f_{r}^{u}(t)$ hold.

Based on the DSO assignment results described in Section 3, we know that at DSO in different time intervals different routes will be used. Thus the day-based incentive routing strategies can be described as follows:

(i) If only one route (i.e., route 2) is used, we do not implement any incentive strategies, just close other routes if necessary ${ }^{3}$.

(ii) If two routes (i.e., routes 2 and 1) are used, users arriving at time $t$ who want to choose route 2 need to pay one Credit $\mathrm{II}^{4}$ for one day. The credits can be obtained by choosing route 1 . Every user can obtain $f_{2}^{s}(t) / f_{1}^{s}(t)$ Credits II if he or she chooses route 1 for one day.

(iii) If all of the three routes are used, users arriving at time $t$ who want to choose route 2 need to pay one Credit II and choose route 1 need to pay one Credit I. The two kinds of credits can be obtained by choosing route 0. Every user can

\footnotetext{
${ }^{3}$ See Proposition 3 for more details.

${ }^{4}$ As mentioned above, in this paper the credits represent one kind of rights. Users can obtain different kinds of rights by choosing different routes, e.g., users can acquire Credits I by choosing route 1 or Credits II by choosing route 2 .
} 
obtain $f_{2}^{s}(t) / f_{0}^{s}(t)$ Credits II and $f_{1}^{s}(t) / f_{0}^{s}(t)$ Credit I if he or she chooses route 0 for one day.

It is worth noticed that the credit is not tradeable in this paper because it is precisely the point of the incentive strategy. Users have first to save credits on non-optimal route to get access to the shortcut. This ensures that a fraction of the flow is move to route 1 and then that the SO can be reached (this is exactly the point achieved with the credit, we know that users will not change otherwise as they would have loose time on a particular day but as they can save on a long run, this choice becomes also efficient at an individual level). All the credit scheme is designed to match the flow switch that is required to move the system from classical UE to SO. Note that we are reasoning on average and we don't specifically care who is using their credits which day.

Before we discuss the effectiveness of the routing strategies, more details about the DSO flow assignment results, especially when two or more routes are used at capacity, need to be given first.

- Type I: DSO flow assignment results are unique when no queue exists or only one route is used; see time intervals $\left(0, T_{2}\right)$ in Fig. $2 ;\left(0, t_{0}\right) \cup\left(T_{1}, T_{2}\right)$ in Fig.3; $\left(0, T_{0}\right) \cup\left(T_{1}, T_{2}\right)$ in Fig. $5 ;\left(0, T_{0}\right) \cup\left(T_{1}, t_{k}\right) \cup\left(t_{k}, T_{2}\right)$ in Fig. $7(\mathrm{~b})$ for example. That is, in these time intervals, the flows arriving at time $t$ will be uniquely assigned to the routes at DSO.

- Type II: DSO flow assignment results are not unique when two or three routes are used at capacity with queues; see other time intervals except those in Type I, i.e., $\left(t_{0}, T_{1}\right)$ in Fig. $3 ;\left(T_{0}, T_{1}\right)$ in Fig. $5 ;\left(T_{0}, T_{1}\right)$ in Fig.7(b).

For Type I, $\lambda_{r}^{s}(t)$ and $f_{r}^{s}(t)=\lambda_{r}^{s}(t) / \lambda(t)$ can be uniquely determined by graphical solutions. For Type II, two extreme optimum solutions for two-route case based on Fig.6 in Muñoz and Laval (2006) are shown in Fig.9. Note that the time interval $\left(t_{1}, T_{1}\right)$ in Fig.9 corresponds to the assignment result of Type II.

Proposition 1. For the assignment solution as shown in Fig.9(a), the trip times of route 2 are always lower than that of route 1 for users arriving at time interval $\left(t_{1}, t^{\star}\right)$. For Fig.9(b), the relationships of trip times between the two routes are not determined for users arriving in time interval $\left(t_{1}, T_{1}\right)$.

Proof. For Fig.9(a), in time interval $\left(t_{1}, t^{\star}\right)$, route 2 is used at capacity with no queues, then $\tau_{2}(t)=\tau_{2}$; route 1 is used at capacity with queues, then $\tau_{1}(t)=\tau_{1}+w_{1}(t)$, where $w_{1}(t) \geqslant 0$. Clearly, we have $\tau_{2}(t)<\tau_{1}(t)$ with the assumption $\tau_{2}<\tau_{1}$. For Fig. $9(\mathrm{~b})$, in time interval $\left(t_{1}, T_{1}\right)$, route 1 is used at capacity with no queues, then $\tau_{1}(t)=\tau_{1}$; route 2 is used at capacity with queues, then $\tau_{2}(t)=\tau_{2}+w_{2}(t)$, where $w_{2}(t)$ is given by

$$
w_{2}(t)=\frac{\int_{t_{1}}^{t}\left(\lambda(t)-\mu_{1}\right) \mathrm{d} t}{\mu_{2}}-\left(t-t_{1}\right), t_{1} \leqslant t \leqslant T_{1}
$$




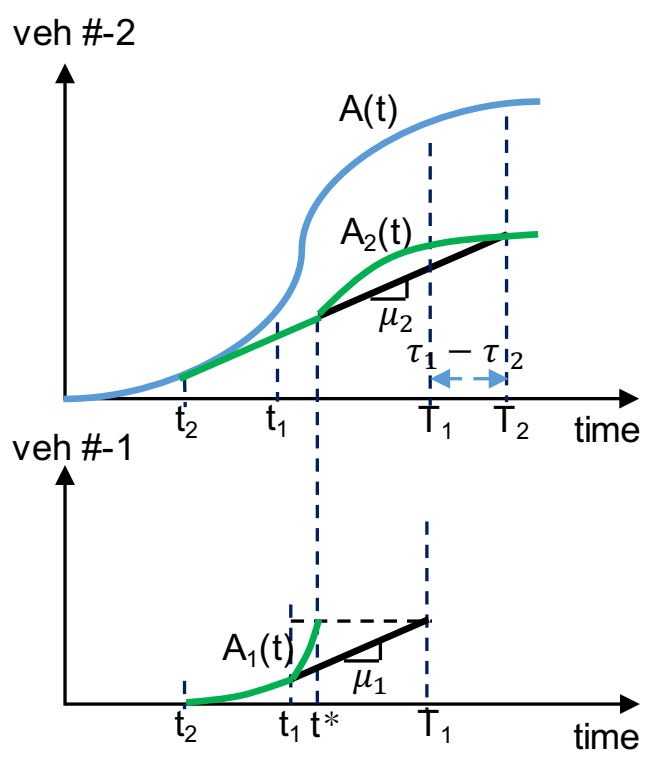

(a) veh \#-2

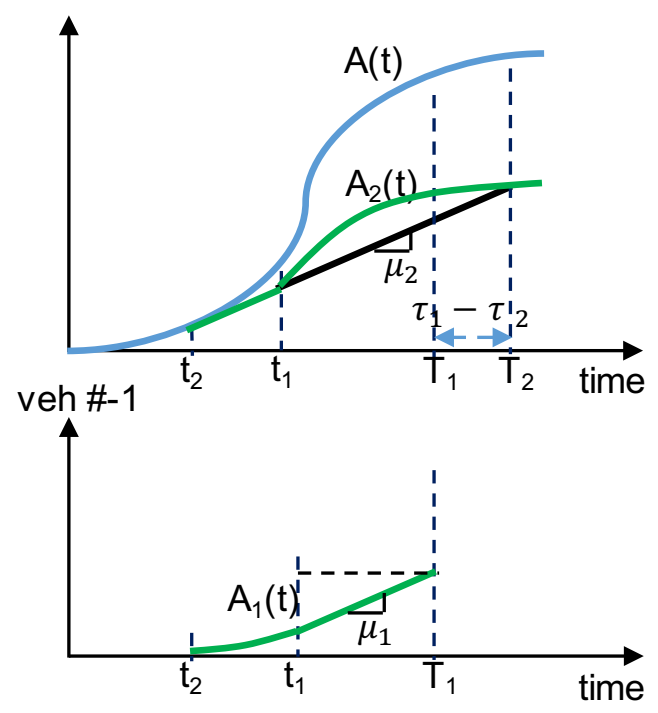

(b)

Fig. 9. Two extreme optimum solutions for two-route case based on Fig.6 in Muñoz and Laval (2006): (a) The queue of route 2 starts as late as possible. (b) No queue grows on route 1.

$$
\dot{w}_{2}(t)=\frac{\lambda(t)-\mu_{1}}{\mu_{2}}-1, t_{1} \leqslant t \leqslant T_{1}
$$

Solve $\dot{w}_{2}(t)=0$, we have $\lambda\left(t_{m}\right)=\mu_{1}+\mu_{2}$, where $t_{m}$ is the moment when $w_{2}(t)$ reaches the maximum value, i.e., $w_{2}\left(t_{m}\right)=\left(A\left(t_{m}\right)-A\left(t_{1}\right)\right) / \mu_{2}-\left(\mu_{1} / \mu_{2}+1\right)\left(t_{m}-t_{1}\right)$. It follows that in time interval $\left(t_{1}, T_{1}\right), w_{2}(t)$ will reach the maximum value, i.e., $w_{2}^{m}$, at time $t_{m}$ or $T_{1}$ due to the unimodal arrival rate. The range of $w_{2}(t)$ is $\left(0, w_{2}^{m}\right)$. When comparing the trip times of two routes based on the solution of Fig.9(b), we can not get the determined relationship. This completes the proof.

Clearly, if the assignment solution as shown in Fig.9(a) is the PUE solution, then we have the fractions of days for all commuters arriving at time $t$ choosing route $r(r=2,1)$ should be the same. This statement can be proved by contradiction. If $f_{1}^{u}(t)_{i}>f_{1}^{u}(t)_{j}$ and $f_{2}^{u}(t)_{i}<f_{2}^{u}(t)_{j}$ hold for User $i$ and User $j$, then they will experience different trip times of total $P$ days based on the Proposition 1. This contradicts the condition of user equilibrium. In all, the conditions, i.e., $f_{2}^{u}(t)_{i}=f_{2}^{u}(t)_{j}=f_{2}^{u}(t)$ and $f_{1}^{u}(t)_{i}=f_{1}^{u}(t)_{j}=$ $f_{1}^{u}(t)$, hold. As a result, the total days that users arriving at time $t$ choose route $r$ for $P$ days are $P \cdot \lambda(t) \cdot f_{r}^{u}(t)$. This means that for each day at PUE the total commuters or flows arriving at time $t($ i.e., $\lambda(t))$ that are assigned to the route $r$ are $\lambda(t) \cdot f_{r}^{u}(t)$. That is, at PUE the fraction of days for each commuter arriving at time $t$ choosing route $r$ and the fraction of total flows arriving at time $t$ that are assigned to the route $r$ for each day are both denoted as $f_{r}^{u}(t)$.

In Proposition 1, it is found that the optimal solution presented in Fig.9(a) has more advantages than Fig.9(b). Another advantage will be shown in proposition 2 below. 
Proposition 2. Considering the DSO flow assignment result presented in Fig.9(a), with strategy (ii), users will spend all of the credits that are obtained by choosing route 1 on route 2 in one period of total $P$ days.

Proof. It is shown in Fig.9(a) that in time interval $\left(t_{1}, t^{\star}\right)$, flows will be assigned to the two routes, though two routes are used at capacity in time interval $\left(t_{1}, T_{1}\right)$. With $t \in\left(t_{1}, t^{\star}\right)$, we have $\lambda_{2}^{s}(t)=\mu_{2}$ and $\lambda_{1}^{s}(t)=\lambda(t)-\mu_{2}$. Correspondingly, $f_{2}^{s}(t)=\mu_{2} / \lambda(t)$ and $f_{1}^{s}(t)=1-f_{2}^{s}(t)$. For each day of total $P$ days, at DSO the fractions of total flows that assigned to the two routes are $f_{2}^{s}(t)$ and $f_{1}^{s}(t)$, respectively.

Due to the lower trip times of route 2 in time interval $\left(t_{1}, t^{\star}\right)$ at DSO as mentioned in Proposition 1, our goal is to prove that, with the strategy (ii), $f_{1}^{s}(t)=f_{1}^{u}(t)$ and $f_{2}^{s}(t)=f_{2}^{u}(t)$ hold. Now consider the strategy (ii) in the following two cases: (1) if $f_{1}^{u}(t)<f_{1}^{s}(t)$, then, $f_{2}^{u}(t)>f_{2}^{s}(t)$ holds. This is because $f_{1}^{u}(t)+f_{2}^{u}(t)=1$ and $f_{1}^{s}(t)+f_{2}^{s}(t)=1$. That is, the PUE fraction of days for each user choosing route 1 is lower than $f_{1}^{s}(t)$, and the corresponding amount of credits obtained by choosing route 1 is $f_{1}^{u}(t) \cdot P \cdot f_{2}^{s}(t) / f_{1}^{s}(t)$, which is lower than $f_{2}^{s}(t) \cdot P$. With these credits users have the right to choose route 2 for one-day use by paying one unit credit, and the fraction of days that users can choose route 2 , i.e., $f_{2}^{u}(t)$, is $f_{1}^{u}(t) \cdot f_{2}^{s}(t) / f_{1}^{s}(t)$. Thus we have $f_{2}^{u}(t)<f_{2}^{s}(t)$. This contradicts the condition $f_{2}^{u}(t)>f_{2}^{s}(t)$. In other words, if one user chooses fewer days in route 1 , he cannot obtain enough credits to choose route 2 in one period. (2) Similarly, if $f_{1}^{u}(t)>f_{1}^{s}(t)$ and $f_{2}^{u}(t)<f_{2}^{s}(t)$ hold, the corresponding amount of credits are higher than $f_{2}^{s}(t) \cdot P$, then we have $f_{2}^{u}(t)>f_{2}^{s}(t)$. This contradicts the condition $f_{2}^{u}(t)<f_{2}^{s}(t)$. In all, at PUE we have $f_{1}^{u}(t)=f_{1}^{s}(t)$ and $f_{2}^{u}(t)=f_{2}^{s}(t)$. This means at PUE for each user arriving at time $t \in\left(t_{1}, t^{\star}\right)$, with routing strategy (ii), the fractions of days in one period of total $P$ days choosing routes 2 and 1 are $f_{2}^{s}(t)$ and $f_{1}^{s}(t)$, respectively. This completes the proof.

In time interval $\left(t_{1}, t^{\star}\right)$, route 2 with lower trip times is more attractive than route 1 , but he or she has to obtain enough credits by choosing route 1 . Proposition 2 states that each user can not reduce his or her total trip times of $P$ days by increasing or decreasing the fraction of days choosing route 1 or 2 . In other words, in this time interval, PUE fractions of total users that are assigned to the two routes at time $t$ for each day are, $f_{1}^{s}(t)$ and $f_{2}^{s}(t)$, respectively. Proposition 2 also states that in time interval $\left(t_{1}, t^{\star}\right)$, the state of DSO can be realized by the strategy (ii).

With the statements of "for each day the fractions of total commuters or flows arriving at time $t$ that are assigned to the route $r$ are same" and "each commuter aims to find the best route on each day", we have the following Proposition 3.

Proposition 3. With the proposed day-based incentive routing strategies, the state of DSO as shown in Fig.9(a) is PUE.

Proof. From Fig.9(a), it is known that the DSO flow assignment results are

1. $0 \leqslant t \leqslant t_{2}$ : all flows are assigned to route 2 .

2. $t_{2} \leqslant t \leqslant t^{\star}$ : assign flows $\mu_{2}$ to route 2 , excess flows $\lambda(t)-\mu_{2}$ are assigned to route 1 . 
3. $t \geqslant t^{\star}$ : all flows are assigned to route 2 .

For $0 \leqslant t \leqslant t_{2}$, it holds $\lambda(t) \leqslant \mu_{2}$, thus no queue exists in this time interval. Choosing route 2 with lower free flow travel time is the optimal choice for each user arriving in this time interval. That is, in $0 \leqslant t \leqslant t_{2}$, DSO is PUE, and no strategies need to be implemented.

For $t_{2} \leqslant t \leqslant t^{\star}$, the time interval $\left(t_{1}, t^{\star}\right)$ has been analyzed in Propositions 1 and 2 , which can be applied to analyze the time interval $\left(t_{2}, t_{1}\right)$. We just need to change the trip times of route 1 to $\tau_{1}(t)=\tau_{1}$ due to the free flow travel time of route 1 in $\left(t_{2}, t_{1}\right)$. In all, in $t_{2} \leqslant t \leqslant t^{\star}$, strategy (ii) needs to be implemented to realize DSO, which is also the state of PUE.

For $t \geqslant t^{\star}$, we just need to close route 1 to make sure only route 2 can be used, which means that route 2 is the optimal choice ${ }^{5}$.

In all, the proposed day-based incentive strategies can realize DSO, which is also the state of PUE. This completes the proof.

Proposition 3 states that one of the DSO flow assignment results as shown in Fig.9(a) can be realized by the proposed day-based incentive strategies. Actually, properties of Propositions 1, 2 and 3 can be easily extended to three-route or general parallel networks; see next Subsections 4.2 and 4.3 for more details.

\subsection{Two examples}

In this subsection, two examples based on Figs.3 and 7(b) will be presented to demonstrate the effectiveness of the proposed day-based incentive routing strategies. In both examples, we first give the extreme DSO flow assignment results based on Fig.9(a), i.e., assign flows as early as possible to the route with higher free flow travel time when routes are used at capacity with queues, and then propose the corresponding strategies.

\subsubsection{Example 1 with Fig.3}

One extreme DSO flow assignment result is shown in Fig.10. Green lines (i.e., $A_{2}(t)$, $\left.A_{1}(t), A_{0}(t)\right)$ represent the cumulative arrival curves of the three routes. $t_{i}$ and $t_{j}$ are uniquely and sequentially determined by

$$
\mu_{0}\left(T_{0}-t_{0}\right)=\int_{t_{0}}^{t_{i}}\left(\lambda(t)-\mu_{2}-\mu_{1}\right) \mathrm{d} t
$$

\footnotetext{
${ }^{5}$ Note that in this time interval $t \geqslant t^{\star}$, the relationships of trip times between the two routes, i.e., $\tau_{2}(t)=\tau_{2}+w_{2}(t)$ and $\tau_{1}(t)=\tau_{1}$, are not determined as shown in Fig.9(a) with the assumption $\tau_{2}<\tau_{1}$. Closing route 1 is one kind of method to make sure the state of DSO. Of course, we can also adopt the toll or credit scheme, but how to use the toll for managers or credit for users is still a question. The user's reluctance to accept tolls is another problem. The value of toll $V$ can be calculated by: (1) determine the two times $t_{u}$ and $t_{v}$ by solving $\tau_{2}(t)=\tau_{1}(t) ;(2)$ in time interval $t_{u} \leqslant t \leqslant t_{v}$, users who want to choose route 1 need to pay toll $V=\tau_{2}(t)-\tau_{1}(t)$ or more. Similarly, we can also calculate the value of credit for the users who want to choose route 2 .
} 


$$
\mu_{1}\left(T_{1}-t_{i}\right)=\int_{t_{i}}^{t_{j}}\left(\lambda(t)-\mu_{2}\right) \mathrm{d} t
$$

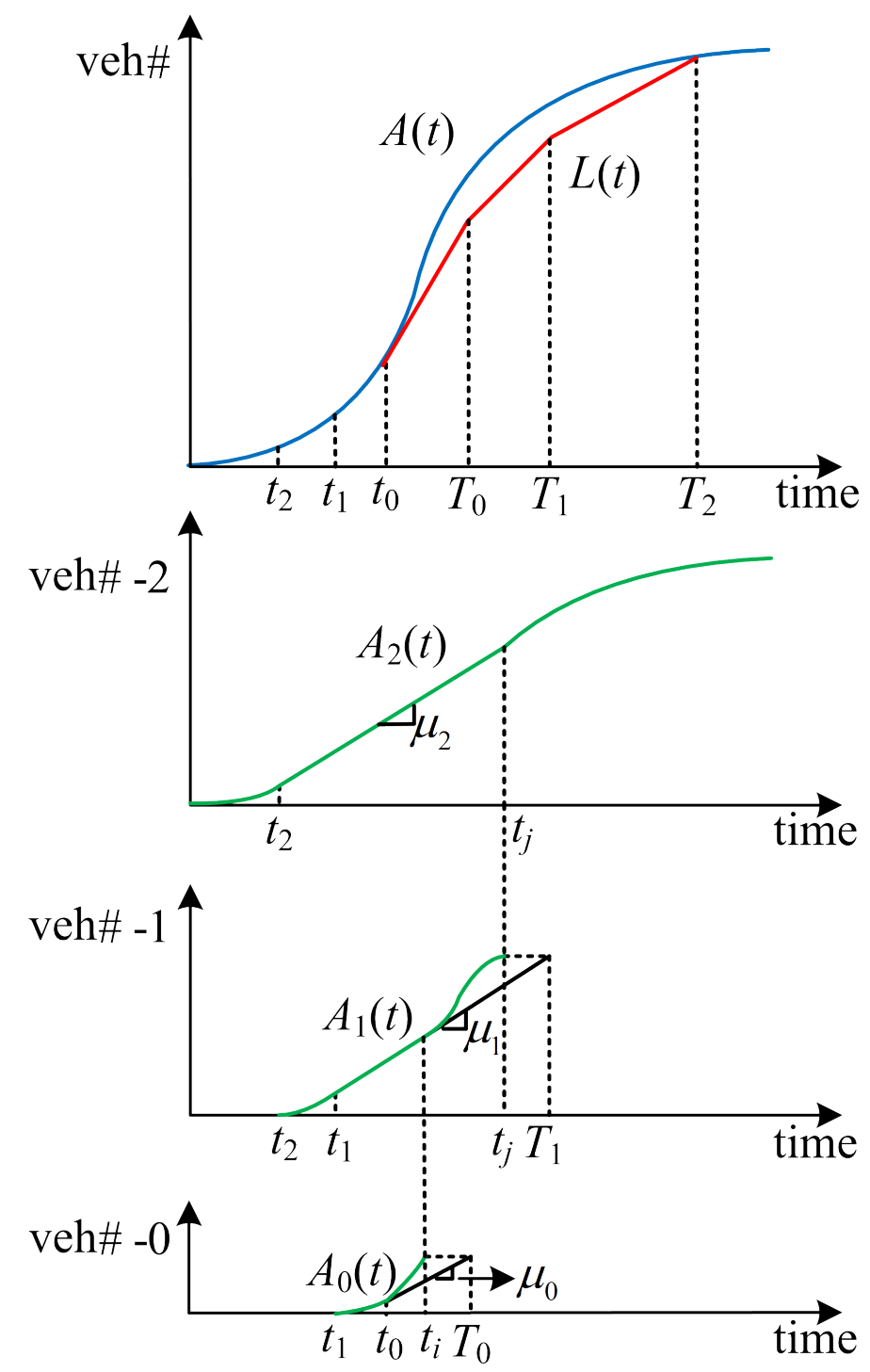

(a)

(b)

(c)

Fig. 10. One extreme DSO flow assignment solution for Case 2.

Based on Fig.10, the DSO flow assignment results are shown in Table 2. The corresponding day-based incentive strategies are:

- In $0 \leqslant t \leqslant t_{2}$, we do not implement any strategies. Because only route 2 is used and has minimal trip times.

- In $t_{2} \leqslant t \leqslant t_{1}$ and $t_{i} \leqslant t \leqslant t_{j}$, each user who wants to choose route 2 needs to pay one Credit II for one day use; $\mu_{2} /\left(\lambda(t)-\mu_{2}\right)$ Credits II can be obtained by choosing route 1 for one day. Based on Proposition 2, we have $f_{2}^{u}(t)=\mu_{2} / \lambda(t)$ 
and $f_{1}^{u}(t)=1-\mu_{2} / \lambda(t)$, which corresponds to the DSO assignment results. Note that, for practical implementation of the scheme, the parameter of period days, $P$, should be given by $P_{\min }=\max \left\{1 / f_{1}^{s}(t) ; 1 / f_{2}^{s}(t)\right\}$, where $P_{\min }$ is the minimal possible value of $P$. In order to ensure the effectiveness of the scheme, the credits obtained in one period of total $P$ days should be spent in the same period. Of course, if the credits can be traded among users, it will become more complicated. We will discuss this topic in the future research.

- In $t_{1} \leqslant t \leqslant t_{i}$, each user needs to pay one Credit II for one day use of route 2 and one Credit I for route $1 ; \mu_{2} /\left(\lambda(t)-\mu_{2}-\mu_{1}\right)$ Credits II and $\mu_{1} /\left(\lambda(t)-\mu_{2}-\mu_{1}\right)$ Credits I can be obtained by choosing route 1 for one day. In this time interval, the relationships of trip times among the three routes at DSO are: $\tau_{2}^{s}<\tau_{1}^{s}<\tau_{0}^{s}$. Thus, Propositions 1,2 and 3 hold in $t_{1} \leqslant t \leqslant t_{i}$.

- In $t \geqslant t_{j}$, close routes 1 and 0 , only route 2 can be used.

Table 2. DSO flow assignment results based on Fig.10

\begin{tabular}{llll}
\hline Time interval & $\lambda_{2}^{s}(t)$ & $\lambda_{1}^{s}(t)$ & $\lambda_{0}^{s}(t)$ \\
\hline $0 \leqslant t \leqslant t_{2}$ & $\lambda(t)$ & 0 & 0 \\
$t_{2} \leqslant t \leqslant t_{1}$ & $\mu_{2}$ & $\lambda(t)-\mu_{2}$ & 0 \\
$t_{1} \leqslant t \leqslant t_{i}$ & $\mu_{2}$ & $\mu_{1}$ & $\lambda(t)-\mu_{2}-\mu_{1}$ \\
$t_{i} \leqslant t \leqslant t_{j}$ & $\mu_{2}$ & $\lambda(t)-\mu_{2}$ & 0 \\
$t \geqslant t_{j}$ & $\lambda(t)$ & 0 & 0 \\
\hline
\end{tabular}

\subsubsection{Example 2 with Fig.7(b)}

Similar with the analysis of Example 1, based on Fig.7(b), the extreme DSO flow assignment results are presented in Fig.11. Green lines, $A_{2}(t)$ and $A_{1}(t)$, represent the cumulative arrival curves of routes 2 and 1 , respectively. $t_{i}$ is uniquely determined by

$$
\mu_{0}\left(T_{1}-T_{0}\right)=\int_{T_{0}}^{t_{i}}\left(\lambda(t)-\mu_{2}\right) \mathrm{d} t
$$

Based on Fig.11, the DSO flow assignment results are shown in Table 3 . The corresponding day-based incentive strategies are:

- In $0 \leqslant t \leqslant t_{2}$, we do not implement any strategies. Because only route 2 is used and has minimal trip times.

- In $t_{2} \leqslant t \leqslant t_{1}, T_{0} \leqslant t \leqslant t_{i}$ and $T_{1} \leqslant t \leqslant t_{k}$, each user who wants to choose route 2 needs to pay one Credit II for one day use; $\mu_{2} /\left(\lambda(t)-\mu_{2}\right)$ Credits II can be obtained by choosing route 1 for one day. 


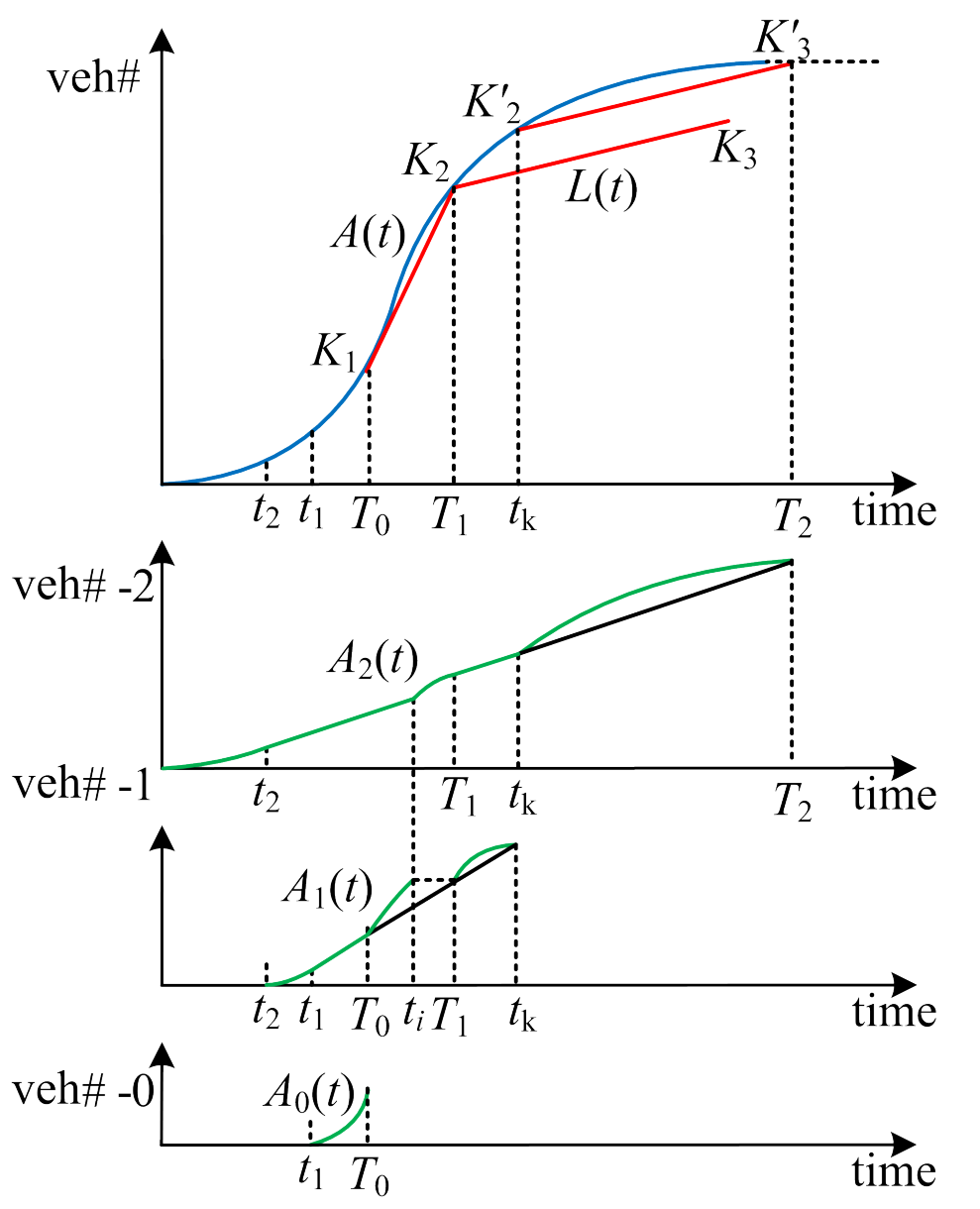

(a)

(b)

(c)

Fig. 11. One extreme DSO flow assignment solution for Case 3b.

- In $t_{1} \leqslant t \leqslant t_{i}$, each user needs to pay one Credit II for one day use of route 2 and one Credit I for route $1 ; \mu_{2} /\left(\lambda(t)-\mu_{2}-\mu_{1}\right)$ Credits II and $\mu_{1} /\left(\lambda(t)-\mu_{2}-\mu_{1}\right)$ Credits I can be obtained by choosing route 1 for one day.

- In $t \geqslant t_{j}, t_{i} \leqslant t \leqslant T_{1}$ and $t \geqslant t_{k}$, close routes 1 and 0 , only route 2 can be used.

\subsection{Extension to general parallel networks}

The extended method to draw DSO allocation lines in this paper can be extended to general parallel networks. Two steps are needed: (1) Sort the routes from low to high according to the size of the free flow travel time, and number the routes as $1,2,3, \ldots r$. Denote $R=\{1,2, \ldots, r\}$. (2) Determine the number of routes, i.e., $n$, that will be used at DSO. It is also assumed that arrival rate is unimodal. Denote the maximum value of $\lambda(t)$ as $\lambda_{m}$. There are two cases to determine $n$. (2a) If there exists $i \in R$, it holds $\lambda_{m}=\sum_{1}^{i} \mu_{r}$. We then have $n=i$. Choose the point $\left(t_{m}, A\left(t_{m}\right)\right)$, where $\lambda\left(t_{m}\right)=$ $\lambda_{m}$, as starting point, draw the allocation lines with slopes $\sum_{1}^{i-1} \mu_{r}, \ldots, \mu_{2}, \mu_{1}$ based on optimality conditions. (2b) If it holds $\sum_{1}^{i} \mu_{r}<\lambda_{m}<\sum_{1}^{i+1} \mu_{r}$, we then have $n=i+1$. 
Table 3. DSO flow assignment results based on Fig.11

\begin{tabular}{llll}
\hline Time interval & $\lambda_{2}^{s}(t)$ & $\lambda_{1}^{s}(t)$ & $\lambda_{0}^{s}(t)$ \\
\hline $0 \leqslant t \leqslant t_{2}$ & $\lambda(t)$ & 0 & 0 \\
$t_{2} \leqslant t \leqslant t_{1}$ & $\mu_{2}$ & $\lambda(t)-\mu_{2}$ & 0 \\
$t_{1} \leqslant t \leqslant T_{0}$ & $\mu_{2}$ & $\mu_{1}$ & $\lambda(t)-\mu_{2}-\mu_{1}$ \\
$T_{0} \leqslant t \leqslant t_{i}$ & $\mu_{2}$ & $\lambda(t)-\mu_{2}$ & 0 \\
$t_{i} \leqslant t \leqslant T_{1}$ & $\lambda(t)$ & 0 & 0 \\
$T_{1} \leqslant t \leqslant t_{k}$ & $\mu_{2}$ & $\lambda(t)-\mu_{2}$ & 0 \\
$t \geqslant t_{k}$ & $\lambda(t)$ & 0 & 0 \\
\hline
\end{tabular}

Choose point $\left(t_{j}, A\left(t_{j}\right)\right)$, where $\lambda\left(t_{j}\right)=\sum_{1}^{i} \mu_{r}$, as starting point, draw the allocation lines with slopes $\sum_{1}^{i} \mu_{r}, \ldots, \mu_{2}, \mu_{1}$ based on optimality conditions. This proposed method to determine $n$ is different with the method mentioned in Muñoz and Laval (2006) (i.e., determine the value of $p$ ).

The day-based incentive routing strategies can also be extended to general parallel networks. With the value of $n$ obtained by the above graphical solution method, the strategies can be described as follows. (i) If only one route, i.e., Route 1, is used, we do not implement any incentive strategies, just close other routes if necessary. (ii) If there are $i, 2 \leqslant i \leqslant n$, routes are used, the users arriving at time $t$ who want to choose Route $j, j=1, \ldots, i-1$, need to pay one Credit $j, j=1, \ldots, i-1$, for one day use. All of these credits can be obtained by choosing Route $j, j=i$. Every user arriving at time $t$ can obtain $f_{j}^{s}(t) / f_{i}^{s}(t), j=1, \ldots, i-1$, Credits $j, j=1, \ldots, i-1$, respectively, if he or she chooses route $j, j=i$, for one day use.

\section{Concluding remarks}

This paper studied the dynamic route choice behavior in a three-route network. With the assumption that cumulative arrival curve is known, we extended the graphical solution method to draw the DSO allocation lines based on point-queue model. The extended method follows that (1) Sort the routes by size in free flow travel time. From low to high, routes should start diverting vehicles and stop diverting from high to low as mentioned in Muñoz and Laval (2006); (2) If there exist routes with infinite capacity, at DSO the vehicles will not be diverted to the routes whose free flow travel time is higher than the first route with infinite capacity; see Cases 1, 1' and 3 for example.(3) If there are no routes with infinite capacity, at DSO three routes will be assigned vehicles; see Case 2 for example. Furthermore, our method is divided into two categories: (i) shift $L(t)$ along $A(t)$ for the case (e.g., Cases 1 and 3 ) that there exist routes with infinite capacity; (ii) shift $L(t)$ along $k_{1}$ for the case (e.g., Cases 2 ) that there is no route with infinite capacity.

Based on the DSO graphical solutions, a simple day-based incentive routing strategy was designed to realize DSO, which provides a benchmark for ITS applications. Some properties were examined base on one extreme DSO flow assignment result as shown 
in Fig.6(a) in Muñoz and Laval (2006).

The framework proposed in this paper can possibly be extended to include (1) multiple-peak demand, (2) tradable credits among users, (3) departure time choice and (4) queue spillovers. Furthermore, a natural extension would be to incorporate the user heterogeneity, e.g., heterogeneous value of trip times. It remains open whether the conclusions obtained in this paper still stand when considering other DSO graphical solutions, e.g., the extreme DSO flow assignment result as shown in Fig.6(b) in Muñoz and Laval (2006). It is worth noticed that our method is not expandable to more general networks but it is important to point out that parallel network like here are very common in practice when studying the morning/evening commute problem, and that the dynamic traffic assignment for general networks is a very challenging problem and many theoretical issues (e.g., existence, uniqueness, stability, solution method) remain to be solved, which is our persistent research objective.

\section{Acknowledgements}

This study has received funding from the European Research Council (ERC) under the European Union's Horizon 2020 research and innovation program (grant agreement No 646592 - MAGnUM project). Chuan-Lin Zhao also acknowledges the funding supported by the National Natural Science Foundation of China(71601012) and The Fundamental Research Funds for Beijing University of Civil Engineering and Architecture(X18148, X18149). We also would like to thank the editor and four anonymous reviewers, especially Reviewer 3, for their valuable comments and suggestions that have improved the quality of this paper substantially.

\section{References}

Abdul Aziz, H.M., Ukkusuri, S.V., 2012. Integration of environmental objectives in a system optimal dynamic traffic assignment model. Computer-Aided Civil and Infrastructure Engineering, 27(7), 494-511.

Akamatsu, T., Wada, K., 2017. Tradable network permits: A new scheme for the most efficient use of network capacity. Transportation Research Part C: Emerging Technologies, 79, 178-195.

Arnott, R., de Palma, A., Lindsey, R., 1990a. Departure time and route choice for the morning commute. Transportation Research Part B: Methodological, 24(3), 209-228.

Arnott, R., de Palma, A., Lindsey, R., 1990b. Economics of a bottleneck. Journal of urban economics, 27(1), 111-130.

Basso, L.J., Jara-Díaz, S.R., 2012. Integrating congestion pricing, transit subsidies and mode choice. Transportation Research Part A: Policy and Practice, 46(6), 890-900.

Carey, M., Watling, D., 2012. Dynamic traffic assignment approximating the kinematic 
wave model: System optimum, marginal costs, externalities and tolls. Transportation Research Part B: Methodological, 46(5), 634-648.

Chen, D., Ahn, S., 2015. Variable speed limit control for severe non-recurrent freeway bottlenecks. Transportation Research Part C: Emerging Technologies, 51, 210-230.

D'Acierno, L., Gallo, M., Montella, B., 2006. Optimisation models for the urban parking pricing problem. Transport Policy, 13(1), 34-48.

Daganzo, C.F., 1995. A pareto optimum congestion reduction scheme. Transportation Research Part B: Methodological, 29(2), 139-154.

Daganzo, C.F., Cassidy, M.J., 2008. Effects of high occupancy vehicle lanes on freeway congestion. Transportation Research Part B: Methodological, 42(10), 861-872.

Daganzo, C.F., Garcia, R.C., 2000. A Pareto Improving Strategy for the TimeDependent Morning Commute Problem. Transportation Science, 34(3):303-311.

Daganzo, C.F., Laval, J.A., Muñoz, J.C., 2002. Ten strategies for freeway congestion mitigation with advanced technologies. Traffic Engineering and Control, 43, 397403.

Daganzo, C.F., Lehe, L.J., 2015. Distance-dependent congestion pricing for downtown zones. Transportation Research Part B: Methodological, 75, 89-99.

Dahlgren, J., 2002. High-occupancy/toll lanes: where should they be implemented? Transportation Research Part A: Policy and Practice, 36(3), 239-255.

Doan, K., Ukkusuri, S.V., 2015. Dynamic system optimal model for multi-OD traffic networks with an advanced spatial queuing model. Transportation Research Part C: Emerging Technologies, 51, 41-65.

Evans, A.W., 1992. Road congestion pricing: when is it a good policy? Journal of Transport Economics and Policy, 26(3), 213-243.

Fosgerau, M., 2011. How a fast lane may replace a congestion toll. Transportation Research Part B: Methodological, 45(6), 845-851.

Friesz, T.L., Luque, J., Tobin, R.L., Wei, B.-W., 1989. Dynamic network traffic assignment considered as a continuous time optimal control problem. Operations Research, 37(6), 893-901.

Ghali, M.O., Smith, M.J., 1995. A model for the dynamic system optimum traffic assignment problem. Transportation Research Part B: Methodological, 29(3), 155170.

Knoop, V.L., Duret, A., Buisson, C., Van Arem, B., 2010. Lane Distribution of Traffic Near Merging Zones - Influence of Variable Speed Limits, Proceedings of the IEEE conference on Intelligent Transport Solutions, September 19-22, Madeira, Portugal

Kuwahara, M., 2007. A theory and implications on dynamic marginal cost. Transportation Research Part A: Policy and Practice, 41(7), 627-643.

Lahlou, S., Wynter, L., 2017. A Nash equilibrium formulation of a tradable credits scheme for incentivizing transport choices: From next-generation public transport mode choice to HOT lanes. Transportation Research Part B: Methodological, 101, $185-212$.

Laval, J.A., 2009. Graphical solution and continuum approximation for the single destination dynamic user equilibrium problem. Transportation Research Part B: 
Methodological, 43 (1), 108-118.

Laval, J.A., Cho, H.W., Muñoz, J.C., Yin, Y., 2015. Real-time congestion pricing strategies for toll facilities. Transportation Research Part B: Methodological, 71, 19-31.

Lu, C.-C., Liu, J., Qu, Y., Peeta, S., Rouphail, N., Zhou, X., 2016. Eco-system optimal time-dependent flow assignment in a congested network. Transportation Research Part B: Methodological, 94, 217-239.

Ma, R., Ban, X. J., Pang, J. S., 2014. Continuous-time dynamic system optimum for single-destination traffic networks with queue spillbacks. Transportation Research Part B: Methodological, 68, 98-122.

Merchant, D.K., Nemhauser, G.L., 1978a. A model and an algorithm for the dynamic traffic assignment problem. Transportation Science, 12, 183-199.

Merchant, D.K., Nemhauser, G.L., 1978b. Optimality conditions for a dynamic traffic assignment model. Transportation Science, 12, 200-207.

Muñoz, J.C., Laval, J.A., 2006. System optimum dynamic traffic assignment graphical solution method for a congested freeway and one destination. Transportation Research Part B: Methodological, 40(1), 1-15.

Nie, Y., 2011. A cell-based Merchant-Nemhauser model for the system optimum dynamic traffic assignment problem. Transportation Research Part B: Methodological, 45(2), 329-342.

Nie, Y., 2017. Why is license plate rationing not a good transport policy? Transportmetrica A: Transport Science, 13(1), 1-23.

Papageorgiou, M., Kotsialos, A., 2002. Freeway ramp metering: an overview. IEEE Transactions on Intelligent Transportation Systems, 3(4), 271-281.

Parry, I.W., Small, K.A., 2009. Should urban transit subsidies be reduced? The American Economic Review, 99(3), 700-724.

Pigou, A.C., 1920. The Economics of Welfare. MacMillan, London.

Rouwendal, J., Verhoef, E.T., Knockaert, J., 2012. Give or take? Rewards versus charges for a congested bottleneck. Regional Science and Urban Economics, 42(1), 166-176.

Shen, W., Zhang, H.M., 2009. On the morning commute problem in a corridor network with multiple bottlenecks: Its system-optimal traffic flow patterns and the realizing tolling scheme. Transportation Research Part B: Methodological, 43(3), 267-284.

Wang, X.L., Ye, H. and Yang, H., 2015. Decentralizing Pareto-efficient network flow/speed patterns with hybrid schemes of speed limit and road pricing. Transportation Research Part E: Logistics and Transportation Review, 83, 51-64.

Yang, H., Huang, H.J., 2005. Mathematical and Economic Theory of Road Pricing. Elsevier.

Yang, H., Wang, X., 2011. Managing network mobility with tradable credits. Transportation Research Part B: Methodological, 45(3), 580-594.

Yang, H., Wang, X.L., Yin, Y., 2012. The impact of speed limits on traffic equilibrium and system performance in networks. Transportation Research Part B: Methodological, 46(10), 1295-1307. 
Ziliaskopoulos, A.K., 2000. A linear programming model for the single destination system optimum dynamic traffic assignment problem. Transportation Science, $34(1), 37-49$.

Zhu, F., Ukkusuri, S. V., 2013. A cell based dynamic system optimum model with non-holding back flows. Transportation Research Part C: Emerging Technologies, $36,367-380$. 